\title{
Anomalous features of the CDW transition in metallic 2H dichalcogenides
}

\author{
C. AYACHE, R. CURRAT* , B. HENNION ${ }^{* *}$ and P. MOLINIE ${ }^{* * *}$ \\ D.R.F.M.C., SPSMS/LCP, CENG, BP. 85X, 38041 Grenoble cedex, France \\ ${ }^{*}$ I.L.L., BP. $156 \mathrm{X}, 38042$ Grenoble cedex, France \\ ** L.L.B., CEN Saclay, 91191 Gif-sur-Yvette, France \\ *** I.M.N., CNRS, 44072 Nantes cedex, France
}

\begin{abstract}
The metallic dichalcogenides of transition metals which crystallise in the trigonal prismatic $2 \mathrm{H}$ structure, are considered as a model family for the study of charge-density-wave transitions of the metal-metal type. We review different properties and their evolution in the series: transport, specific heat, magnetic susceptibility, soft phonon dynamics. For temperatures higher than the transition temperature, $T_{d}$ many characters underline the departure from the normal metal behaviour as well as from the behaviour resulting from the presence of the simple soft mode predicted by weak-coupling theories. A phenomenological analysis rather suggests for this family a very strong coupling mechanism involving local vibronic pseudo spins, narrow-band character and proximity to an order-disorder transition.
\end{abstract}

\section{Introduction.}

Metallic dichalcogenides of transition metals, $\mathrm{MX} 2(\mathrm{M}=\mathrm{Nb}, \mathrm{Ta} ; \mathrm{X}=\mathrm{S}, \mathrm{Se})$, which crystallise in the trigonal prismatic $2 \mathrm{H}$ lamellar structure are well-known for undergoing charge-densitywave (CDW) transitions linked to their two-dimensional character [1-3]. The CDW transition temperatures, $\mathrm{T}_{\mathrm{d}}$, are $123 \mathrm{~K}, 80 \mathrm{~K}$ and $33 \mathrm{~K}$ for $\mathrm{TaSe}_{2}, \mathrm{TaS}_{2}$ and $\mathrm{NbSe}_{2}$, respectively, while $\mathrm{NbS}_{2}$ undergoes no phase transition. The accompanying incommensurate lattice distortion which also develops below $\mathrm{T}_{\mathrm{d}}$ is however close to the commensurate modulation wave-vector $Q=\left(a^{*} / 3,0,0\right)$. The modulation is indeed triple- $Q$ and the displacements of ionic centres belong to the $\Sigma_{1}$ symmetry ( this predominantly consists in a longitudinal motion of the cations). The preceding characters are shared by all the compounds undergoing the CDW transformation. Continuous efforts in structural studies over many years revealed also a variety of additional features $[4,5]$ which confirm the intimate similarities existing between these compounds.

The mechanism of the transition is thought to mainly result from the electron-phonon interaction in presence of quasi-2D "nesting" conditions. The different band structure calculations [6] for these systems agree about a Fermi Surface made of quasi-cylindrical pockets, the first ones elongated along $\mathrm{HKH}$ edges and the last one with its axis along АГА in the Brillouin zone. A van Hove anomaly resulting from the connection between the zone centre isoenergy surfaces and the edges ones is also possible but its distance from the Fermi level, $\varepsilon_{\mathrm{F}}$, is still uncertain. The total width of the predominantly $\mathrm{d}_{\mathrm{z}} 2$ character band near $\varepsilon_{\mathrm{F}}$ is more than $2 \mathrm{eV}$. A detailed theoretical investigation of the electron-phonon interaction in those systems was carried out by MOTIZUKI and co-workers [7] on-the basis of an effective tight binding model. This study show the importance of both the nesting conditions and of the q-dependent 
electron-phonon coupling coefficient for causing the lattice instability. This approach, a weak coupling one in essence, obtained good success in particular by predicting the right symmetry for the soft phonon mode. However it failed to provide a satisfactory evaluation of the different observed $T_{d}$ 's. This can be due to limits in the calculation but most probably results from more basic reasons involving the need for a short coherence length model [8] and a strong coupling approach [9]. The strong coupling nature of the present CDW's received renewed support recently owing to the use of STM spectroscopy which indicates very large ratios between the CDW gap and $\mathrm{T}_{\mathrm{d}}: 2 \Delta / \mathrm{kT}_{\mathrm{d}}=15.2,15.4$ and 23.9 for $\mathrm{TaSe}_{2}, \mathrm{TaS}_{2}$ and $\mathrm{NbSe}_{2}$, respectively [10]. These values remain however much lower than the I.R. reflectivity data [11]: 46 in the case of $\mathrm{TaSe}_{2}$. In the following, we review selected experimental topics which could help for a better understanding of the CDW formation in this model family.

\section{Macroscopic properties.}

At first sight, transport properties of $2 \mathrm{H}-\mathrm{MX} \mathrm{X}_{2}$ compounds appear to follow closely the normal metal behaviour. This contrasts with the 1T-polytypes for which case the CDW transition is of the metal-non metal type. In $2 \mathrm{H}$ systems both the distorted and the undistorted phase, above and below $T_{d}$, are fully metallic. At lower temperatures, the resistivity $\mathrm{Q}(\mathrm{T})$ behaves like $\mathrm{T}^{3}$ or $\mathrm{T}^{5}$ for $\mathrm{Nb}$ and Ta compounds respectively, as expected for $\mathrm{d}$ metals [12]. For distorted systems, but not in $\mathrm{NbS2}$, a large positive magneto resistance is observed below $\mathrm{T}_{\mathrm{d}}$ which is due to magnetic breakdown effects in the reconstructed band structure [13]. Near $T_{d}$, a small anomaly can be detected in $\varrho(T)$ as a result of the CDW pseudo-gap formation which is confirmed by the strong increase in the absolute value of the Hall coefficient, $R_{\mathrm{H}}[12]$. Above $\mathrm{T}_{\mathrm{d}}$ however, several difficulties raise in comparison with the above normal aspects. The first problem concerns the resistivity of $\mathrm{TaSe}_{2}$ and, to a less extent of $\mathrm{TaS}_{2}$, for which an additional constant contribution is present above $T_{d}$. The presence of a correlated minimum in the phonon dominated thermal conductivity is strongly suggestive of a pseudo-spin mechanism for scattering both electrons and phonons [14]. Transport experiments can be then accounted for by an additional constant relaxation rate which is progressively reduced as the CDW order sets in below $T_{d}$. The analysis of the specific heat supports the proposed scheme which basically represents the CDW transition as an order-disorder one [14,15]. The form of the specific heat anomaly near $\mathrm{T}_{\mathrm{d}}$ in $\mathrm{TaSe}_{2}$ is well accounted for by a jump contribution plus a gaussian fluctuation one reflecting the short coherence length $\left(\xi_{0}-10 \AA\right)$ [16]. This was considered by McMILLAN as an argument in favour of a "local soft mode" [8]. Our approach goes one step further by considering the order-disorder character of the transition which agrees well with the variation of the transition entropy $\Delta S \# R \ln 2$, once one refers to the unit cell of the distorted phase i.e. nine times that of the high temperature crystallographic phase. This view implies that the local soft mode has not a continuous character but it is rather of a pseudo-spin nature with a double degeneracy. A detailed picture of the atomic displacements and of the accompanying CDW modulation was proposed [14].

However, the above picture breaks down for the other compounds in the series. First, the transition entropy becomes significantly less than the order-disorder value. Instead, it scales with the transition temperature [15]. Secondly, a refined investigation of $\mathrm{Q}(\mathrm{T})$ shows the existence of "saturation" effects, i.e. a negative curvature, occurring in the higher temperature range. This effect is most pronounced for $\mathrm{NbS}_{2}$. This behaviour is not fortuitous as shown by the thermoelectric power, S [15]. The latter has negative values at room temperature for all compounds. This contradicts the positive Hall effect and the exclusively positive charge carriers predicted by band calculation. In addition, large absolute values are achieved 


\section{TABLE I}

\begin{tabular}{|c|c|c|c|c|}
\cline { 2 - 5 } \multicolumn{1}{c|}{} & $\mathrm{NbS}_{2}$ & $\mathrm{NbSe}_{2}$ & $\mathrm{TaS}_{2}$ & $\mathrm{TaSe}_{2}$ \\
\hline $2 \gamma$ (in K) & 570 & 770 & 820 & 830 \\
\hline$\delta \varepsilon_{0}$ (in K) & 75 & 57 & 51 & 13 \\
\hline $2 \Delta$ (in K) & - & 790 & 1160 & 1850 \\
\hline
\end{tabular}

especially for NbS2 which exceeds $20 \mu \mathrm{VK}^{-1}$ at room temperature and this is inconsistent with a normal metal behaviour. The contradiction can be overcome by assuming a resonant level structure close to $\varepsilon_{\mathrm{F}}$ by analogy with the case of mixed-valence systems. Then, the S-vs-T curves above $T_{d}$ can be easily fitted and provide a quantitative estimate for the width, $2 \gamma$, and for the shift relative to $\varepsilon_{\mathrm{F}}, \delta \varepsilon_{0}$, of this structure [15]. The corresponding results are shown in TABLE I. These show that, excepting NbSe2, the widths are of similar magnitude for the other compounds and that differences between them mainly come from differences in position relatively to the Fermi level. We also note that this phenomenological treatment is also able to explain consistently the excess departure of the Wiedeman-Franz ratio from the Lorentz number [15]. Interestingly, this structure is also reflected in the magnetic susceptibility which shows a departure from the usual Pauli paramagnetism. In Figure 1, the normalised curves for the different compounds nearly coincide above $T_{d}$. In this range, using the first and second derivatives of the density of states, $v(\varepsilon), \chi$ can be expanded as $\chi \propto v\left(\varepsilon_{F}\right)+1 / 6 \pi^{2}\left(v^{\prime \prime}\left(\varepsilon_{F}\right)-v^{\prime 2}\left(\varepsilon_{F}\right) / v\left(\varepsilon_{F}\right)\right)\left(k_{B} T\right)^{2} \approx v\left(\varepsilon_{F}\right)+1 / 6 \pi^{2} T^{2} / T_{0}^{2}$. From the data of

Figure 1, one obtains $\mathrm{T}_{0} \sim 700 \mathrm{~K}$, a value which agrees well with $2 \gamma$. In Table $I$ we have also reported the CDW pseudo-gap values obtained from STM [10]. These values are of the order of magnitude of $2 \gamma$ though generally higher, especially in the case of $\mathrm{TaSe}_{2}$. This may be due to the way the pseudo-gap is defined in presence of dispersion effects. If confirmed, this relative agreement would suggest that the CDW gap formation is limited by a narrow-band structure near $\varepsilon_{\mathrm{F}}$. This could also constitutes an issue for explaining why $\chi$ does not reduce when crossing $\mathrm{T}_{\mathrm{d}}$ contrary to what is observed for Ta-compounds (see Figure 1 ).

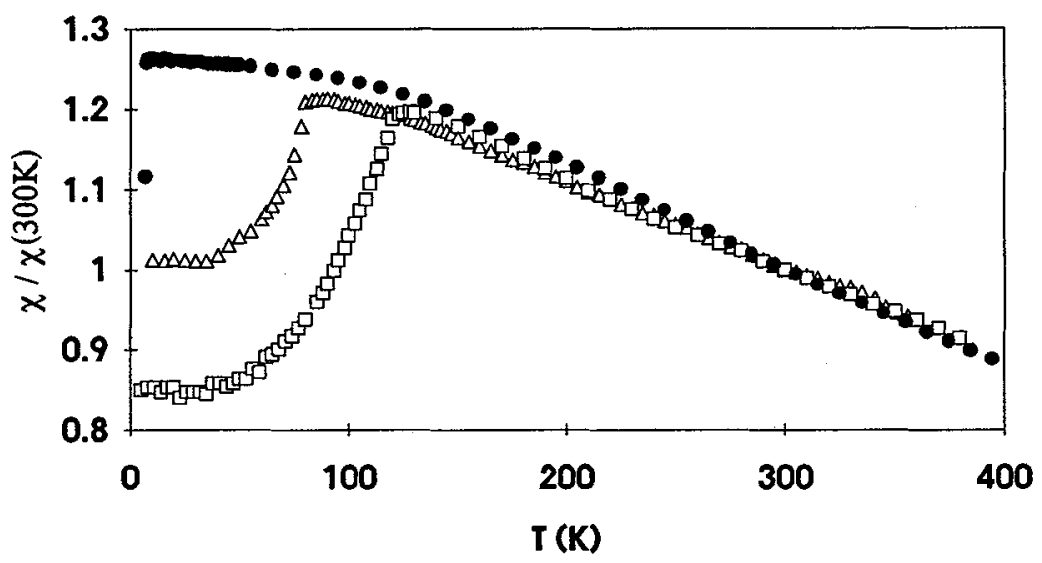

FIGURE 1. Magnetic susceptibility of $\mathrm{TaSe}_{2}$ (squares), $\mathrm{TaS}_{2}$ (triangles) and $\mathrm{NbSe}_{2}$ (circles), normalised at $\mathrm{T}=300 \mathrm{~K}$. 


\section{Inelastic neutron diffusion.}

The above phenomenological approach seems to provide a satisfactory representation of macroscopic quantities. However, it has to be supported by more microscopic evidence. This motivated our reinvestigation of the soft phonon dynamics by measuring inelastic diffusion of neutrons using different diffractometers at ILL and LLB. We are mainly focusing on the study of $\mathrm{NbSe}_{2}$ and $\mathrm{TaSe}_{2}$.

MONCTON and co-workers [4] have measured the room temperature dispersion curve of the mode $\Sigma_{1}$. This possesses a peculiar form with a broad dip centred around $Q=\left(a^{*} / 3,0,0\right)$, the position of the over-structure wave-vector in the reciprocal lattice. Our investigations fully confirm this result at a quantitative level. However, the energy scans in the vicinity of $Q$ show that, despite improved resolution conditions, the broad soft phonon oscillator is possibly mixed with an even broader structure. In the case of $\mathrm{NbSe}_{2}$, we measured the softening of the Q-phonon down to $\mathrm{T}_{\mathrm{d}}$ and showed that the latter is complete within experimental accuracy [16]. However, two modes with the $\Sigma_{1}$ symmetry seem present at least in the lower temperature range: $\omega_{1}$, the higher energy one and, $\omega_{2}$, the lower energy one. Then, analysing the energy scans for all temperatures on the basis of two oscillator contributions, an anomalous temperature dependence is obtained for $\omega_{2}$. The latter is roughly constant between $100 \mathrm{~K}$ and $300 \mathrm{~K}$, while, below $100 \mathrm{~K}$, it decreases more rapidly than the expected $\omega_{2}^{2} \propto\left(T-T_{d}\right) \omega_{2}^{2} \propto\left(T-T_{d}\right)$ law. $\omega_{1}$ does not change significantly with temperature but its value is determined with a large uncertainty. Recently, we confirmed the different behaviour of the mode softening which occurs in $\mathrm{TaSe}_{2}$ [17]. This concerns particularly the presence of a gap at $\mathrm{T}_{\mathrm{d}}$ in the $\omega_{\mathrm{Q}}(\mathrm{T})$ curve as first obtained in Ref.[4]. No extra oscillator seems to soften unlike the case of $\mathrm{NbSe}_{2}$. Instead, a broad contribution is present and is well represented by a lorentzian shape. The confirmation of a gap in the $\omega_{\mathrm{Q}}(\mathrm{T})$ curve, supports the order-disorder mechanism for the transition in $\mathrm{TaSe}_{2}$ as this behaviour is predicted by theoretical models [18] which, in addition, predict a complex inelastic structure. On the contrary, NbSe2 looks shifted towards the displacive regime. However, the complex structure still shown by the inelastic response indicates the departure from the purely displacive case. Definite conclusions concerning this point have also to consider the specific features of the $2 \mathrm{H}$ structure like interaction of different modes of $\Sigma_{1}$ symmetry or $2 \mathrm{D}-3 \mathrm{D}$ dimensionality crossover.

1. WILSON J.A. and YOFFE A.D., Adv.Phys. 18 (1969) 193.

2 WILSON J.A., DiSALVO F.J. and MAHAJAN S., Adv.Phys. 24 (1975) 117.

3. FRIEND R.H. and JEROME D., J.Phys.C12 (1979) 1441.

4. MONCTON D.E.,AXE J.D. and DiSALVO F.J., Phys.Rev.B16 (1977) 801.

5. FUNG K.K., MCKERNAN S., STEEDS J.W. and WILSON J.A., JPhys.C14 (1981) 5417; BIRD D.M., MCKERNAN S., STEEDS J.W. and WITTHERS R.L. J.Phys.C18 (1985) 499.

6. MATTHEIS L.F. Phys.Rev. B8 (1973) 3719; WOOLLEY A.M. and WEXLERG., J.Phys.C10 (1977) 2601.

7. MOTIZUKI K. and SUZUKI N. in Structural Phase Transitions in Layered Transition Metal Compounds, K.MOTIZUKI ed. (Reidel, Dordrecht,1986).

8. MCMILLAN W.L, Phys.Rev.B16 (1977) 643.

9. VARMA C.M. and SIMONS A.L., Phys.Rev.Lett.51 (1983) 138.

10. HESS H.F. et al., J.Vac.Sci.Technol.A8 (1990) 450; WANG C., SLOUGH C.G. and COLEMAN R.V., J.Vac.Sci.Technol.B9 (1991) 1048.

11. BARKER A.S., DITZENBERGER J.A. and DiSALVO F.J., Phys.Rev.B12 (1975) 2049.

12. NAITO M. and TANAKA S., J.Phys.Soc.Japan 51 (1982) 219.

13. NAITOM. and TANAKA S., J.Phys.Soc.Japan 51 (1982) 228.

14. NUNEZ-REGUEIRO M.D., LOPEZ-CASTILLOO J.M. and AYACHE C., Phys.Rev.Lett.55 (1985) 1931.

15. AYACHE C. et al., in Recent Advances in Magnetism of Transition Metal Compounds, A.KOTANI and N.SUZUKI eds.(World Sc.Publ.Co.), 1993, p.241.

16. AYACHE C., CURRAT R. and MOLINIE P., Physica B $180 \& 181$ (1992) 333.

17. AYACHE C., CURRAT R., B.HENNION and MOLINIE P., unpublished.

18. AUBRY S., J.Chem.Phys.62 (1975) 3217. 